\title{
An Automatic Monitoring Method of Slope Deformation in Open-pit mine based on BP Neural Network and GIS Technology
}

\author{
Yan-Jun He ${ }^{1}$, Tao Chen ${ }^{2}$ and Liu Han ${ }^{2}$ \\ ${ }^{1}$ Mining Research Institute, Inner Mongolia University of Science and Technology, Baotou, China \\ ${ }^{2}$ College of Mines, China University of Mining \& Technology, Xuzhou, China \\ Received: April 14, 2020. Revised: May 20, 2020. Accepted: June 19, 2020. Published: July 20, 2020.
}

\begin{abstract}
At present, the research on BP neural network has achieved good results in many industries and fields, but there are few projects in the application research of mineral resources mining. Under the social background of the rapid development of electronic information technology, BP neural network and GIS technology are combined to carry out research and application, which will provide a new research path for slope deformation monitoring and disaster prevention in mining area. Therefore, in the paper, the key technology of open-pit mine slope deformation automatic monitoring based on BP neural network and GIS technology was put forward. Firstly, the advantages of BP neural network were analyzed and BP neural network was selected as the prediction model of slope deformation. The artificial fish swarm algorithm was used to improve the BP neural network to improve the performance of the model. Based on the analysis and construction of GIS technology, the combination application of BP neural network and GIS technology was discussed. Through practice, the application effect of the technology was verified, and it has good theoretical and practical value.
\end{abstract}

Keywords-BP neural network, GIS technology, slope deformation, automatic monitoring, key technology

\section{INTRODUCTION}

$\mathrm{U}$ NDER the background of the great economic development of the country, the scale, depth and scope of various open mineral projects in China have gradually increased [1]. The open-pit mine is affected by various factors, such as slope design, natural geography and geological conditions, human construction and construction. And landslides, collapses and other accidents often occur during construction. These accidents not only directly lead to production delay or stop production, but also bring many casualties, and cause very bad negative effects in society. Detecting the slope of open-pit mine and studying the inherent deformation rule of slope can effectively reduce the occurrence frequency of instability, collapse and other accidents caused by slope deformation [2]. In the past, the measurement of slope deformation in China mainly adopts the geodetic method, which has the characteristics of technical specifications and simple operation. However, the traditional measurement methods mainly represented by manual measurement are often affected by the surrounding geological environment of the open pit, and the monitoring workload is heavy. The monitoring data can't be transmitted in time, and the use effect is not good in the comprehensive research and effective use of data [3]. Moreover, the monitoring accuracy of this measurement method is not high, which may directly affect the judgment of slope stability and lay a hidden danger for the accident. Therefore, the electronic information technology and database technology are used to monitor the slope deformation comprehensively. The stress deformation and trend of the side slope of open pit mine are controlled dynamically in real time. And the nonlinear artificial intelligence algorithm, neural network and other mathematical models are used to automatically monitor the slope deformation of open-pit mine, which has important theoretical value and popularization and application significance [4].

In the planned economy period of the $60 \mathrm{~s}$ of last century, the research on Open-pit Slope in China was limited to the mechanism, distribution law and trend prediction of disaster formation. Attention is paid to the formation and activity of slope hazards. Most of the methods used are qualitative analysis [5]. The results of this study often have no scientific basis for disaster analysis, leading to the slow development of slope disaster evaluation and research theory. After the reform and opening up, the state has increased the exploitation and utilization of various mineral resources [6]. The research on the slope deformation monitoring technology of open-pit mine has also been paid attention to and developed. On the basis of introducing and drawing on the international advanced experience, the theories of statistics, geography, meteorology, environment and mathematics have been applied in China. The quantitative and semi quantitative evaluation of slope deformation monitoring in open-pit mine are conducted, and good research results are obtained. [7]. Since entering the new century, in the supporting of the development of electronic 
information, computer database and mathematical model analysis technology, the study is carried out from qualitative analysis to quantitative evaluation, from simple factor analysis to comprehensive evaluation of total factor analysis, from the traditional manual measurement and drawing of electronic information and computer measurement database, and the study expands the network technology development, which have promoted the rapid development and progress of monitoring of open-pit mine slope deformation [8]. In this paper, based on BP neural network algorithm and GIS technology, the mathematical model of slope deformation in open-pit mine was established, and the application characteristics of the model were verified by an actual monitoring case in combination with an automatic monitoring system [9].

\section{BP NEURAL NETWORK MODEL AND OPTIMIZATION ALGORITHM FOR SLOPE DEFORMATION AUTOMATIC MONITORING}

\section{A The Principle of Artificial Neural Network}

The study of neural networks began in the 40s of last century, mainly focused on the physiology and psychology of brain function, and the electrophysiological aspects of neurons [10]. American psychologist Mika Rocco and mathematician Peters jointly established the mathematical model of formal neurons in 1943, which laid the foundation of neuroscience theory research [11]. In the following 20 years, many scientists have studied this theory from different angles, such as neuronal link strength, artificial intelligence, neural networks and so on, and obtained many important research results [12]. But research has entered the bottleneck stage, which leads to the neural network has been in the trough in the research of new analog computer and artificial intelligence technology in the last century before 80 s [13]. In 1982, American physicist Hope Fede put forward the HNN model, and introduced the concept of computational ability function so that the neural network stability research has a digital model that can be based on, as shown in Fig.1. The neural networks are used successfully to solve the ancient traveling salesman problem, and it is also a landmark research result in the history of artificial neural network research since modern times [14]. In 1986, Lu Mei Hart and Maclachlan proposed a BP algorithm to solve the weight correction of multilayer neural networks. This neural network algorithm, also known as back propagation learning algorithm, is widely used in many fields such as automatic control, language research and so on [15].

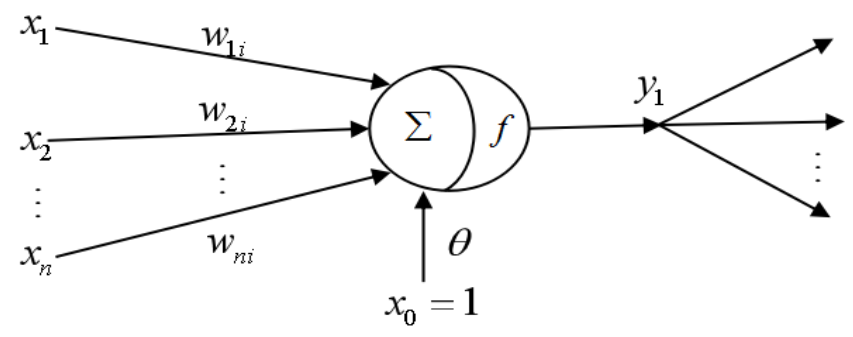

Fig.1 Neuron structure model

Neural network, as an abstract mathematical model, uses a large number of neural nodes to reflect the structure and function of human brain. This complex neural network can simulate human learning, memory, reasoning, computing and other behaviors [16]. The artificial neural network adopts inductive learning method, and uses large-scale empirical research to study again and again. In the process of internal adaptation, the weights of each neuron are modified so that the mutual structure and the connection weight distribution of the neural network are stable. In this way, the whole process of acquiring knowledge and learning is expressed. The most important feature of neural network is that it has nonlinear, adaptive and powerful learning function and error correction function. This is also consistent with the complexity and nonlinear characteristics of slope stability, so the study of slope deformation has a good basis for mathematical model research [17]. As shown in figure 1. The most basic single neuron structure model in neural networks is a nonlinear node form from multiple inputs to one output [18]. The input and output relations can be represented by formula (1), where $x_{j}(j=1,2, \cdots, n)$ is the signal that is transmitted from other neural cells. B represents the threshold value, and $\mathrm{C}$ indicates the weight of the connection between the cell $\mathrm{j}$ and the cell $\mathrm{i}$.

$$
\begin{aligned}
I_{i} & =\sum_{j=1}^{n} w_{j i} x_{j}-\theta_{i} \\
y_{i} & =f\left(I_{i}\right)
\end{aligned}
$$

$f(\cdot)$ is a transfer function, and it can be either a linear function or a nonlinear function with arbitrary order derivatives. The commonly used function types are jump order function, sigmoid function and Gauss function, which are shown in formula (2), (3) and (4). These three transfer functions also correspond to the three basic hierarchical structures of feed-forward, feedback and self-organized competition of artificial neural networks At present, the most widely used three kinds of neural network models are BP network, Hopfield network and Kohonen network. Among them, BP neural network development is the most mature, and are the most widely used and stable [19].

$$
\begin{gathered}
f(x)= \begin{cases}1 & x \geq 0 \\
0 & x<0\end{cases} \\
f(x)=\operatorname{th}(x)=\frac{e^{x}-e^{-x}}{e^{x}+e^{-x}} \\
y_{i}=\exp \left(-\frac{1}{2 \sigma_{i}^{2}} \sum_{j}\left(x_{j}-w_{j i}\right)^{2}\right)
\end{gathered}
$$

\section{B BP Neural Network Algorithm}

BP neural network is information feed-forward layered neural network, which has the typical characteristics of error back propagation, and shows bidirectional propagation in the learning algorithm. Its network structure is divided into input layer, output layer and hidden layer. The hidden layer here may be one layer or multiple layers, and each layer will link forward through the connection weights between nodes. 
After a signal input, the BP neural network will transmit the signal to the hidden layer. After the excitation function is calculated, the information is transmitted to the output layer, and then changes to the output signal. The training process of BP neural network is characterized by two kinds of propagation: forward and reverse. When the signal takes the process of "input layer- hidden layer- output layer", it shows the state of forward propagation. When the hidden layer accepts the signal and produces an error between the output signal and the error and the error exceeds the expected range, the system will correct the weights and thresholds of each layer according to this error, and make the layers become more adaptive so as to improve the performance of the system. This reaction is in a state of reverse propagation. The structure of the BP network model is shown in Fig.2. A single node represents a neuron in a network. There is no connection between neurons in the same layer, and each layer of the node only accepts the input of the previous layer node. The output of each neuron only affects the nodes of the subsequent output layer. For a generally simple data set, one hidden layer is usually sufficient. The deeper the number of layers, the more overfitting problems may be caused. At the same time, it will increase the difficulty of training and make the model difficult to converge. However, for complex data sets involving time series or computer vision, additional layers are required, and multiple hidden layers can be used to fit nonlinear functions. The number of hidden layers and the effect/use of the neural network can be summarized as follows: Hidden layers=1: Can fit any "continuous mapping from one finite space to another finite space" function; Hidden layers $=2$ : With an appropriate activation function, it can represent any decision boundary of arbitrary precision, and can fit any smooth mapping of any precision; the number of hidden layers $>2$ : The additional hidden layers can learn complex descriptions.

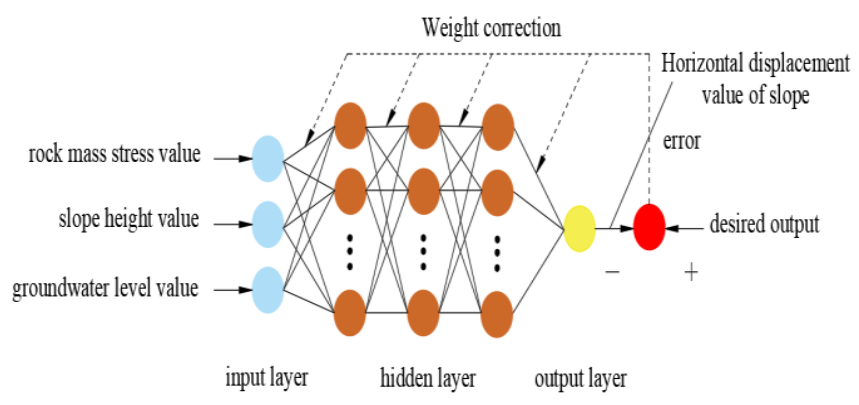

Fig.2 Structure of BP neural network

The basic algorithm of BP neural network generally adopts the learning mode of teachers. The main steps of its basic learning algorithm are as follows.

Step 1. The initialization settings of weights $w$ and $\theta$ are carried out, and the connection weight $w_{i j}$ of the input layer to the hidden layer neuron is determined. The connection weight between the hidden layer and the output layer is $w_{j k}$ and the threshold of the hidden layer is set to $\theta_{j}$. The threshold $\theta_{k}$ for the output layer neuron is given a smaller value between $(0,1)$.
Step 2. The input vector $x_{i}=\left(x_{1}, x_{2}, \cdots, x_{m}\right)$ is determined, and the corresponding expected output vector is $\hat{Y}_{i}=\left(\hat{Y}_{1}, \hat{Y}_{2}, \cdots, \hat{Y}_{n}\right)$. The value of $x_{i}$ is input to the neuron node of the input layer. Forward calculation is carried out according to $x_{j}^{i}=f\left(\sum_{i=0}^{n} W_{i j} x_{i}-\theta_{j}\right) \quad(j=1,2, \cdots, u)$, or reverse calculation is carried out based on $y_{k}=f\left(\sum_{k=0}^{n} V_{j k} x_{j}-\theta_{k}\right) \quad(k=1,2, \cdots, n)$.

Step 3. The error difference between output neuron output value and expected output value is calculated. If the error difference is in line with expectations, the training is over. If the gap is too large, it will re-enter the reverse calculation link of the model calculation. (4) After repeated correction function calculation, the weight to meet the requirements is got. The model is finished and the output of the signal is output. Algorithm flow chart of BP neural network learning is shown in Fig.3.

\section{COMBINATION OF BP NEURAL NETWORK AND GIS TECHNOLOGY IN AUTOMATIC MONITORING OF SLOPE DEFORMATION}

\section{A Construction of GIS System}

The full name of the GIS system is called "geographic information system", which is a kind of computer system. The system is mainly used to collect and store, analyze and detect, describe the application and spatial location, geographic location related information of a computer operating system. The objects processed and managed by GIS system mainly include all kinds of geospatial data. These data mainly include spatial location parameters, remote sensing graphic information and entity attribute data. Through the powerful calculation and analysis processing function, the various phenomena and processes in the region are studied. On the basis of analysis and calculation, some suggestions for management decision are put forward. The system can not only realize the processing of digital and text information by ordinary computer data system, but also has good spatial modeling ability. After acquiring a lot of related information, it constructs a geographic analysis model which can meet the requirements of professional research, comprehensive analysis and integrated processing, and has the ability of cross analysis of multi space. This can help researchers discover the deep connection between space from the phenomenon of distribution, and it is an important assistant and staff to deal with complex planning and to carry out numerous and complicated research. The content system of the GIS system is shown in Fig.4.

GIS system mainly includes spatial overlay analysis, terrain and geomorphology analysis, 3D spatial analysis and other functions. Spatial overlay analysis can manage spatial database hierarchically. In the topological analysis of a particular target, a new data layer is added to the data overlay, and the mathematical attributes are added to further determine the spatial relationship of the entity attribute. 
Topographic analysis is mainly used to calculate slope, aspect and other parameters in slope deformation. This can not only output digital form analysis, but also generate slope and aspect distribution map. This can achieve automatic and effective factor evaluation, and reduce the influence of human factors. Three-dimensional spatial analysis can realize the visual performance of open-pit mine in the system, and presents the characteristics of topography and geomorphology from different perspectives. After comprehensive processing with other thematic maps, the image can be realized with realistic and virtual view.

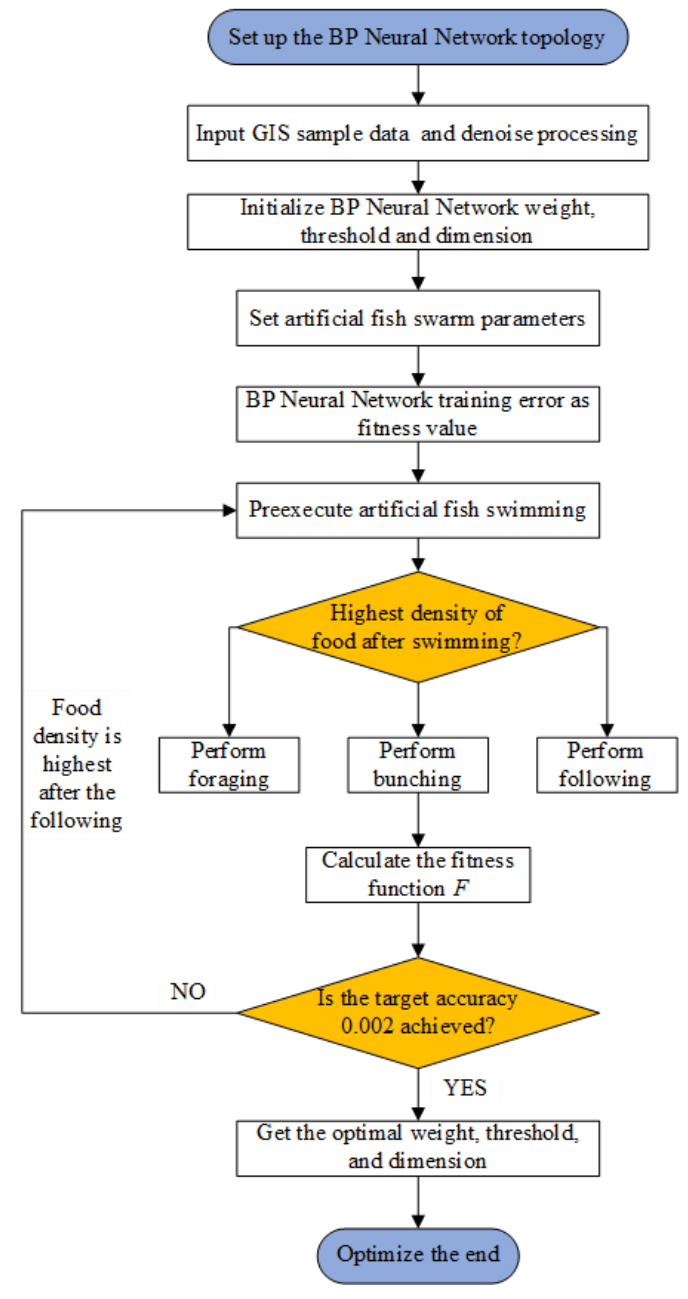

Fig.3 BP neural network learning algorithm flow chart



Fig.4 GIS system content architecture

GIS full relational database has 4 advantages. The relation of attribute data, geometric data and graphic image data can be realized. The spatial data, attribute data and graphic data can be extracted and used without the establishment of complex connections, and the data processing is quick. In the traditional data management mode, DBMS manages the attribute data and file system manages inconsistent spatial data management. Through the complex construction in the process of data development, the simple and unified operation of the whole database to the spatial data is realized. The system meets the industry standard and has reliable safety performance. GIS full database system has good development, and can use a variety of hardware platforms and operating systems. Data format is in line with industrial standards, which is conducive to the promotion and data sharing in different enterprises. The system has automatic recovery function, security privilege management mechanism and security warning function. This ensures the safe and reliable use of GIS spatial data system. The architecture of the GIS fully relational database system is shown in Fig.5.

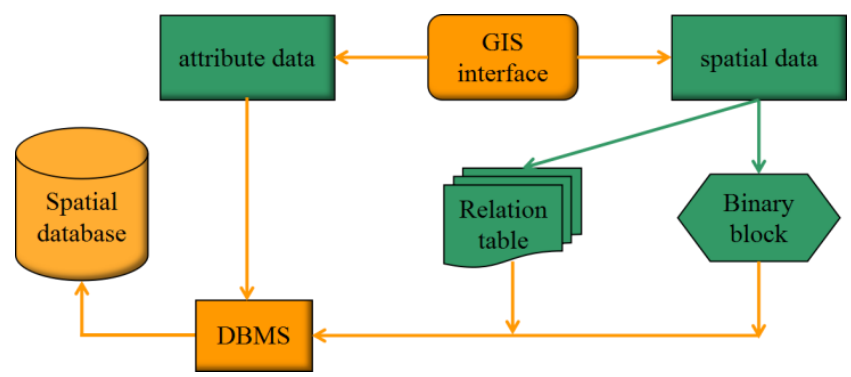

Fig.5 GIS full relational database system diagram

\section{$B$ The Combination of BP Optimization Algorithm and GIS System}

BP neural network is used to determine weights and thresholds in a random way, and it is easy to cause over-fitting phenomenon. When the number of nodes in the neural network structure is determined by the experts, it is easy to bring about the influence of human factors. All these will lead to inaccurate data analysis in BP neural networks. The results are not true. Therefore, in this paper, the artificial fish swarm algorithm was used to optimize the BP neural network, which was based on the global optimization function of artificial fish swarm algorithm to obtain the initialization weights and thresholds of the optimal state, and to build a better network structure. The main steps of using artificial fish swarm algorithm to optimize the structure of BP neural network are as follows. In the definition of artificial fish individual, $\mathrm{D}$ is set to represent the total number of units in $\mathrm{BP}$ neural network, and $\mathrm{D}=\mathrm{p}$.

The state vector of artificial fish is regarded as the attribute vector of hidden layer, and the distance between two artificial fish is calculated by $d_{a, b}=\sum_{i=1}^{N}\left(x_{i}^{a}(t)-x_{i}^{b}(t)\right)^{2}$, and the adaptive function is $F=c_{1} \cdot \frac{q}{Q}+c_{2} \cdot \frac{1}{M S E}$ Among them, $c_{1}$ and $c_{2}$ are equilibrium constants, and $q$ is the total number of units in the initial hidden layer, and $Q$ is the total number of units in the residual hidden layer. An artificial fish represents a BP neural network, and the difference between the two artificial fish also represents a BP neural network. In 
the optimization process, the state of the hidden unit is determined by whether the state of the artificial fish is larger than the threshold of the hidden layer attribute. If the state value $x_{i}^{a}(t)_{(i=1,2, \cdots, D)}$ is greater than the threshold of the hidden layer zodiac, retain the corresponding hidden units ate retained. If it is not greater than that, the implied unit and the attached weights and thresholds are deleted. Then the artificial fish is trained to calculate the fitness value of each artificial fish, and the optimal number of hidden units is found in the continuous search. In view of the improved artificial fish swarm algorithm (AFSA) BP neural network prediction model, compared with the traditional BP neural network, the training speed and network prediction of the network are better. The effect can be seen from contrast map between the measured displacement and the AFSA-BP model prediction, which is shown in Fig.6.

The BP neural network optimized by artificial fish swarm algorithm is used to monitor the slope deformation of open-pit mine. The influence factors of slope deformation are taken as input, the nonlinear network model of the deformation value of open-pit slope is taken as the output. A large number of observation data are needed as training samples to establish training network so as to achieve the purpose of monitoring the deformation of the slope. Data samples mainly come from data collection of GIS system. In the GIS system, the data collected from the input layer mainly include the average slope of the surface, the slope of the leading edge, the average inclination of the screen, the maximum monthly rainfall, the recent signs of activity and the construction engineering activities. And they are considered as the contents of GIS spatial database to be classified. GIS spatial database system is a hierarchical model database, which divides data into one to many structure relations. The capacity and content of the database are expanded according to the increase of the number of layers. In the spatial database system, it is mainly divided into data layer, business layer and presentation layer. The architecture of GIS spatial database system is shown in Fig.7. The underlying data layer is the base of the database system, where the GPS data is managed by SQL Server, and data analysis is carried out according to historical results to provide technical support for data analysis of software systems. The intermediate business layer is responsible for processing the system data and producing the analysis and early warning data to meet the needs of the operators. In open-pit mine slope monitoring system, the basic data and conversion format are generally accepted. The GPS monitoring data are collected and analyzed, and the slope development of mining area is predicted.



Fig.6 Comparison of measured displacement and AFSA-BP model prediction

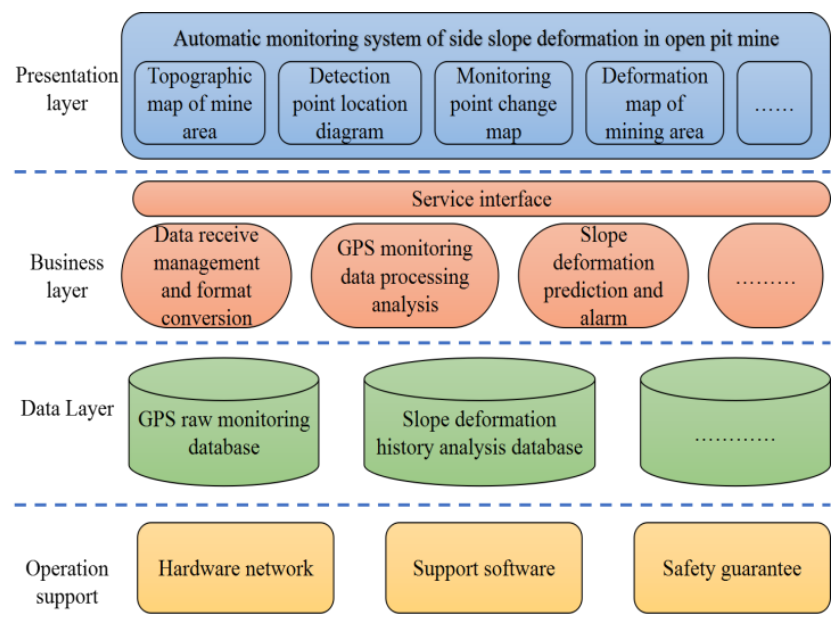

Fig.7 GIS spatial database system diagram

In the combination of BP neural network and GIS system, after determining the influencing factors and completing the basic data collection, the relative weight of various factors in the slope deformation system should be considered, and the scientific choice should be taken to meet the requirements of the accuracy of the mathematical model calculation. Mining of GIS system get the slope of the space geometric data (slope angle, slope height, etc.), based on the monitoring data of GIS system, input the fish swarm algorithm optimized BP Neural Network training and data fitting, predict the slope settlement and horizontal slippage, the relation between geometric deformation space on the map, which according to set value on the slope sliding to forecast in advance. The causes of slope deformation in open pit mine are complex, and have the characteristics of diversity, nonlinearity and randomness. Therefore, in this paper, combined with fuzzy matter element theory and analytic hierarchy process, quantitative evaluation of factors affecting slope deformation was carried out. When choosing and evaluating the evaluation factors, it is necessary to try to make each factor independent of each other and not affected by their own. In factor selection, all factors that affect the slope deformation are taken into full consideration from the aspects of topography, engineering geology and so on. At the same time, according to the actual situation to choose and accept, evaluation index with relatively lean and valuable is selected to ensure the effect of evaluation. 


\section{NETWORK AND GIS SYSTEM}

In this paper, based on BP neural network and GIS system technology, an automatic monitoring system model of slope deformation was established. Combined with the example of M mountain open-pit coal mine slope in M Province, the application of slope deformation monitoring and early warning based on monitoring analysis was carried out so as to test the performance of the model, as shown in Fig.8. The open pit coal mine is a large open pit mine in China, which is a combination of hills and depressions. Its top slope height has reached more than 200 meters and it is a typical high steep slope. The monitoring of the slope deformation mainly retains the stress monitoring of the slope rock mass, slope deformation monitoring and water flow monitoring. In order to improve the accuracy of deformation monitoring, 24 hours uninterrupted dynamic monitoring is realized. GPS deformation monitoring technology is introduced to realize the monitoring of deformation and stress.

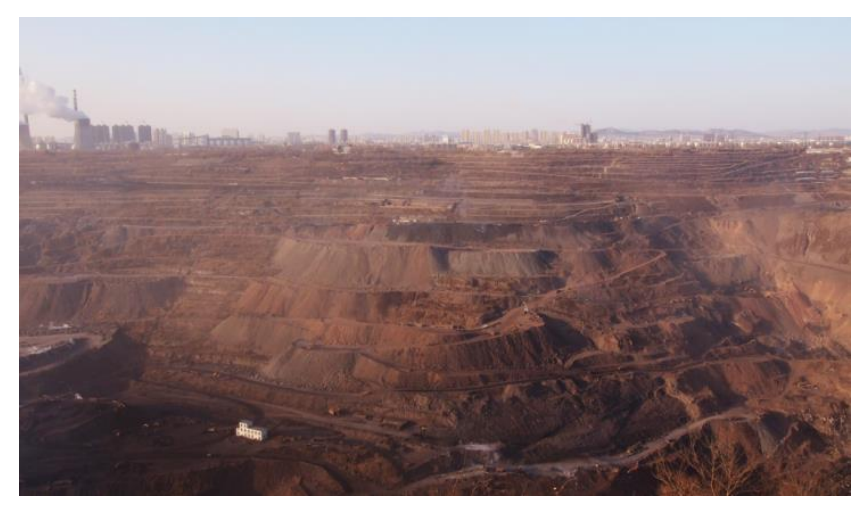

Fig. $8 \mathrm{M}$ mountain open pit coal mine in M Province

Matlab 2018 is used for data fitting training on a computer with a dual-core CPU and 16G RAM. Parameter setting in artificial fish school algorithm: artificial fish quantity Fish Num=10; maximum setting period $T \max =50$; maximum number of trials Try Num $=1$; crowding factor $\delta=0.618$; initial visual field $\mathrm{Fv}=1.5$; visual walking coefficient $\lambda=0.2$. There are three main stopping criteria for the neural network, which are set to the maximum number of Epoch: 1000; Performance: 0.002; Validation checks: 20 times, and the remaining criteria are the default values, such as infinite iteration Time, Gradient 1e-7, etc. .In the slope displacement monitoring of open-pit coal mine, the sample data processing should be carried out first, and the mathematical analysis model is established. Long term monitoring can collect a large amount of data on slopes, but these data are not all valid. Therefore, the wavelet data analysis toolbox is needed to de-noise the original data of slope. Then the data of training samples are determined. The slope deformation monitoring model based on BP neural network is established. The GIS system can monitor the parameters (including the stress condition of the rock mass, the change of the slope height, the groundwater level of the slope and the slope horizontal displacement). A total of 180 sets of sample data, of which 150 sets are used for data training of BP neural network, and 30 sets of sample data are used to verify the accuracy of training. In this paper, the input layer contents of the neural network are set to the stress condition of the rock mass, the change of the slope height and the groundwater level of the slope. The output layer is set as the displacement value in the horizontal direction of the slope. 180 sets of data are selected from the original data as training samples of BP neural network model. Considering the difference of input data samples and in order to avoid the difference between the absolute value of input quantity is too large so that the BP neural network appears over-fitting, the sample data are normalized before the BP neural network starts training. The input value can be balanced between [-1, 1]. The normalized formula of the original sample data is $x=2 \times \frac{x-x_{\text {min }}}{x_{\max }-x_{\min }}-1$. Among them, $x_{\max }$ and $x_{\text {min }}$ represent the maximum and minimum values when the sample is input. After the initialization design of the BP neural automatic monitoring model, the artificial fish swarm algorithm is used to select samples for learning and training. $x_{i}^{a}(t)$ is a state value of the s artificial fish, which may be a weight or a threshold value. Each artificial fish can represent a BP Neural Network, including the ownership value and threshold of BP Neural Network, so it is called Artificial Fish Neural Network. Artificial fish can evaluate the current BP Neural Network error and simulate the following, bunching and foraging behavior. Each artificial fish selects swimming behavior and compares the concentration of food after swimming to select the best swimming behavior. The selection of artificial fish swimming behavior is based on the maximum food density after selecting the behavior. The default behavior is foraging behavior, and if the other two behaviors are not satisfied, foraging behavior is performed. When all artificial fish in swimming, the fitness function $\mathrm{F}$ will be calculated automatically, and then the food density of artificial fish will be checked whether it reaches the preset value (goal=0.002). If it reaches the preset value, the optimal weight, threshold and dimension value will be obtained. If not, the cycle will continue. With the optimization of parameters by the fish swarm algorithm, the BP Neural Network model is constantly optimized, so that the optimal fitness value is constantly adjusted during the training process. First establish the initial BP neural network, use the network weights, thresholds and dimensional parameters as the position of the individual fish, set the parameters of the artificial fish school, and use the fitness function as the target value. Through the predation, grouping and trailing behavior of the fish school Adjust the position of the fish school, use the fish school algorithm to obtain the characteristics of the global optimal solution, and continuously adjust the weights and thresholds of the neural network to achieve the smallest error between the output value and the target value. This optimization process can avoid the defect of the traditional BP neural network falling into the local optimal solution.At the end of the training, the optimal fitness is output. It can be seen from the training chart of BP neural network in Fig.9 that the number of hidden layer units determined by BP neural network is 8 . It also indicates that the BP neural network is successful in monitoring the displacement and deformation of the slope.

Based on the GIS system, the risk assessment of landslide hazard in open-pit coal mine is carried out. It is based on the output data of BP neural network. Spatial overlay analysis of GIS is used to establish a special map for influencing factors 
of landslide in opencast coal mine. The base of all kinds of factors are made spatial overlay in order to generate a distribution map of landslide hazard. Then, the special auxiliary analysis to the slope, the aspect and the elevation from GIS is used to make a dominant macroscopic qualitative analysis to the danger which possibly appears in the open pit coal mine. Fig.10 is a landslide hazard assessment map generated by the GIS system. The danger degree of landslide is represented by different colors and numerical values. The bigger the number, the more dangerous the representation is.

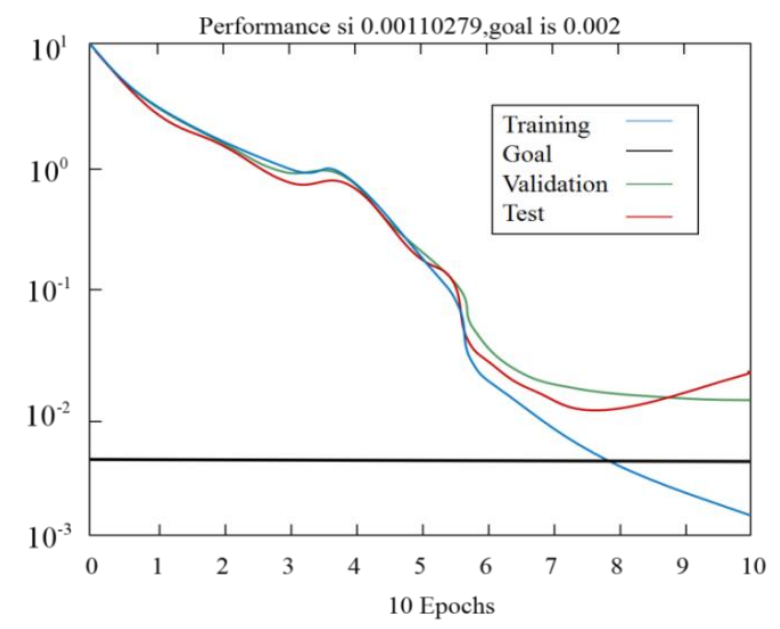

Fig.9 BP neural network training effect diagram

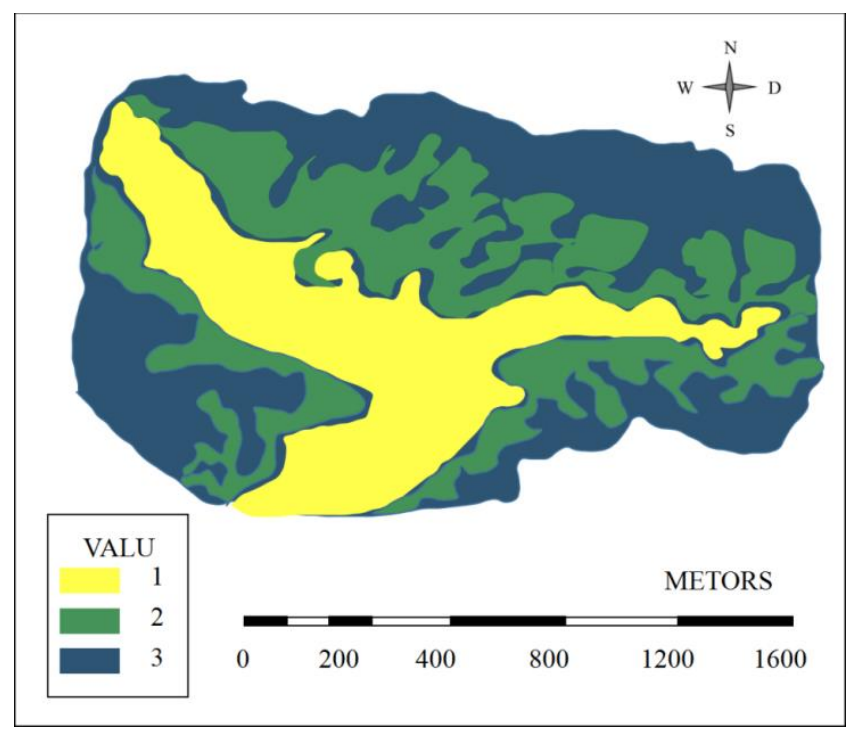

Fig.10 Landslide hazard assessment map

The risk distribution of the open pit mining in different areas can be visually seen through landslide hazard assessment map of open-pit mine slope generated by GIS system. After the managers find the dangerous area, the optimized BP neural network model is used to calculate the quantitative migration of the landslide in the dangerous area so as to provide a scientific basis for the next step. The automatic monitoring system of open-pit slope can produce various charts such as landslide hazard assessment map. Real time positioning and monitoring are carried out to slopes. At the same time, the development trend and harm of slope are analyzed according to the mathematical model. Based on the comprehensive application of GIS technology and BP neural network, a new and effective way for the automatic monitoring of slope deformation was provided in this paper, which had good popularization and application value.

\section{CONCLUSIONS}

The nonlinear and adaptive characteristics and the strong learning error correction function of BP neural network can effectively solve the complex and nonlinear problems encountered in the cause of slope stability. Therefore, based on BP neural network, the advantages of GIS technology are used to carry out research, which can help slope hazard research into a scientific, digital, refined stage. The traditional BP neural network has the defects of slow convergence and convergence to local optimal values, which is extremely unfavorable for slope monitoring and early warning. When the neural network converges to the local optimal solution, it may cause prediction failure, causing casualties and property. loss. The fish swarm algorithm uses global optimization, and the global optimal solution is selected from multiple local optimal solutions, so it has the characteristics of quickly obtaining the global optimal value. Combining the two algorithms, under the condition of the same number of iterations, a high-precision neural network can be obtained, thereby obtaining an automatic monitoring method of open-pit mine slope deformation based on BP neural network and GIS technology. But the method proposed in this paper is based on a large number of data samples, and has high requirements on the amount of data from the slope monitoring source, which needs to be provided by the GIS system.

In this paper, according to BP neural network and GIS technology, the automatic monitoring technology of slope deformation in open-pit mine was studied. Specific analysis of BP neural network model was carried out, and the advantages of GIS technology and system construction were discussed. The BP neural network model was used to calculate the slope displacement, and the development trend was also studied and prediction information was provided. After using artificial fish algorithm to optimize the BP neural network, the optimal network structure was found, which provided good data support for the GIS system. GIS technology was used to analyze the slope hazard caused by the output value of BP neural network, such as slope displacement data. The landslide hazard distribution map and other analysis charts were generated to provide scientific decision-making basis for management. In this paper, the automatic detection technology of slope deformation based on BP neural network and GIS technology was applied in M open-pit coal mine of $M$ Province, and the real-time monitoring of slope displacement was realized. Slope hazard distribution diagram generated by the system and other charts has a strong guiding role.

In the future, further optimization of the calculation steps and accuracy of BP Neural Network is the key to improve the sensitivity of slope monitoring and early warning. The combination of $5 \mathrm{G}$ technology, cloud computing, BP Neural Network and GIS technology is conducive to real-time transmission, processing and monitoring of slope deformation data, which is conducive to the realization of intelligent monitoring and early warning system and the construction of intelligent mine cloud platform. 


\section{ACKNOWLEDGEMENT}

This work was financially supported by the National Natural Science Foundation of China (No. 51804298, No. 51774271).

\section{REFERENCES}

[1] Suh J, Choi Y, and Park H D., "GIS-based evaluation of mining-induced subsidence susceptibility considering 3D multiple mine drifts and estimated mined panels," Environmental Earth Sciences, vol.75, pp. 1-19, 2016.

[2] Zhang X L, Jun H U, and Zhao T Y., "Geometric Parameters Optimization of Dump Slope Based on BP Neural Network-Genetic Algorithm," Mining \& Metallurgical Engineering, vol. 66, pp. 630-644, 2017.

[3] Hejmanowski R, and Witkowski W T., "Suitability assessment of artificial neural network to approximate surface subsidence due to rock mass drainage," Journal of Sustainable Mining, vol. 14, pp.101-107, 2015.

[4] Chok Y H, Jaksa M B, and Kaggwa W S, "Neural network prediction of the reliability of heterogeneous cohesive slopes," International Journal for Numerical \& Analytical Methods in Geomechanics, vol.40, pp.1556-1569, 2016.

[5] Chen S Y, Zhou C B, and Jiang N, "Blasting Vibration Velocity Prediction of Open-pit to Underground Mining Slope," Blasting, vol.25, pp.729-741, 2015.

[6] Luma A, and Anwar A H., "Salmonellainfections modelling in Mississippi using neural network and geographical information system (GIS)," Bmj Open, vol. 6, pp.88-97, 2016.

[7] Wu H, Huang C, and Zhang J, "Deformation monitoring system for high slope in open pit mine with the integration of GNSS and GIS," Wuhan Daxue Xuebao, vol. 40, pp.706-710, 2015.

[8] Chen W, Pourghasemi H R, and Zhao Z., "A GIS-based comparative study of Dempster-Shafer, logistic regression, and artificial neural network models for landslide susceptibility mapping," Geocarto International, vol. 32, pp.367-385.2016.

[9] Pham B T, Bui D T, and Pourghasemi H R, "Landslide susceptibility assesssment in the Uttarakhand area (India) using GIS: a comparison study of prediction capability of naïve bayes, multilayer perceptron neural networks, and functional trees method,' Theoretical \& Applied Climatology, vol.122, pp.1-19, 2015.

[10] Erzin Y, and Ecemis N., "The use of neural networks for CPT-based liquefaction screening," Bulletin of Engineering Geology \& the Environment, vol. 74, pp.103-116, 2015.

[11] Zeng B, Xiang W, and Rohn J, “Assessment of shallow landslide susceptibility using an artificial neural network in Enshi region," Natural Hazards \& Earth System Sciences Discussions, vol. 54, pp.1-46, 2017.

[12] Khakestar M S, Hassani H, and Moarefvand P, "Prediction of the collapsing risk of mining slopes based on geostatistical interpretation of geotechnical parameters," Journal of the Geological Society of India, vol. 87, pp.97-104, 2016.

[13] Chen W, Li W, and Hou E, "RETRACTED ARTICLE: GIS-based landslide susceptibility mapping using analytical hierarchy process (AHP) and certainty factor (CF) models for the Baozhong region of Baoji City, China," Environmental Earth Sciences, vol. 73, pp. 3951-3951, 2015.

[14] Bui D T, Ho T C, and Pradhan B, "GIS-based modeling of rainfall-induced landslides using data mining-based functional trees classifier with AdaBoost, Bagging, and MultiBoost ensemble frameworks," Environmental Earth Sciences, vol. 75, pp. 1-22, 2016.

[15] Benzer S, Benli Ç K, and Benzer R., 'The comparison of growth with length-weight relation and artificial neural networks of crayfish, Astacus leptodactylus, in Mogan Lake," Journal of the Black Sea/mediterranean Environment, vol. 59, pp. 187-202, 2015.

[16] Wang Q, Li W, and Xing M, "Landslide susceptibility mapping at Gongliu county, China using artificial neural network and weight of evidence models," Geosciences Journal, vol. 20, pp. 1-14, 2016.

[17] Sheikhian H, Delavar M R, and Stein A., "A GIS- based multi- criteria seismic vulnerability assessment using the integration of granular computing rule extraction and artificial neural networks," Transactions in Gis, vol. 21, pp. 2624-2629, 2017.

[18]Kumar K, and Annadurai R., "Landslide Susceptibility Mapping Using Analytical Hierarchy Process and Artificial Neural Network at Kothagiri Taluk, India,' International Journal of Earth ences and Engineering, vol. 8, pp. 775-787, 2015.

[19] Hashemi S A, and Kashi H., "Determination of number of check dams by artificial neural networks in arid regions of Iran," Water Science \& Technology A Journal of the International Association on Water Pollution Research, vol.72, pp. 952, 2015.

\section{Creative Commons Attribution License 4.0 (Attribution 4.0 International, CC BY 4.0)}

This article is published under the terms of the Creative Commons Attribution License 4.0 https://creativecommons.org/licenses/by/4.0/deed.en_US 